\title{
NEUROCISTICERCOSE EM PACIENTES INTERNADOS POR EPILEPSIA NO HOSPITAL REGIONAL DE CHAPECÓ REGIÃO OESTE DO ESTADO DE SANTA CATARINA
}

\author{
PAULO CESAR TREVISOL-BITTENCOURT*, NONOHAI CUNHA DA SILVA**, \\ ROBERTO FIGUEREDO***
}

\begin{abstract}
RESUMO - Neurocisticercose é condição provocada pela infecção do sistema nervoso pelo Cysticercus cellulosae, a forma larvária da Taenia solium. Ela é, muito provavelmente, a principal responsável pela elevada prevalência de epilepsia no Brasil. Em diversas áreas do país, possui um caráter endêmico, gerando grande dispêndio financeiro para um enfrentamento das diversas complicações neurológicas relacionadas a ela. Sabe-se que por suas peculariedades sócio-econômicas, com suinocultura disseminada, a região sul do Brasil, é considerada pelo ministério da Saúde como uma área de alto risco. Para averiguar sua importância entre nós, um estudo, considerando internações por epilepsia e sua etiologia, foi realizado na cidade de Chapecó, principal cidade do oeste de Santa Catarina. O período coberto pela investigação foi de 1995, entrada em funcionamento de tomógrafo computadorizado no Hospital Regional daquela cidade, até o mês de novembro de 1996. Uma expressiva prevalência de neurocisticercose, aproximada de $24 \%$, foi observada entre os indivíduos internados pelo diagnóstico de epilepsia. Algo em torno de $40 \%$ dos infectados apresentava lesões em sua fase ativa, sugerindo uma aquisição recente. Nossos dados sugerem que a despeito desta região sediar agro-indústrias de projeção internacional, a cisticercose grassa endemicamente na área. Um controle deste flagelo terceiro mundista poderia ser obtido com a difusão de mensagens educativas, em linguajar apropriado, para uma sensibilização de toda a população vivendo naquela região. Além disso, todos aqueles indivíduos envolvidos em suinocultura, independentemente de como seja esta classificada, deveriam ser objeto de atenção especial.
\end{abstract}

PALAVRAS-CHAVES: neurocisticercose, epilepsia, suinocultura.

\section{Prevalence of neurocysticercosis among epileptic in-patients in the west of Santa Catarina - southern Brazil}

ABSTRACT - Neurocysticercosis (NC) is an endemic condition in several areas of Brazil. It is most likely the major responsible for the high prevalence of epilepsy in our country, estimated in $1-2 \%$ of general population. We performed a study to evaluate NC as aetiology of epilepsy in the west of Santa Catarina. This state belong to southern Brazil and it has a very developed economy. However, due the widespread swine farming in the west district, many of them without any sanitary control, the national health authorities have considered all this area at risk to NC. The study was carried out in Chapecó, the main town in that region, where CT Scan service was started in 1995. All patients put on hospital care due epileptic seizures in 1995-96 were considered. Febrile convulsions were excluded of the sample. We found a very expressive prevalence rate of NC among patients suffering from epilepsy. Roughly $24 \%$ of these patients, showed unequivocal tomography evidences for the diagnosis of NC. Our data suggest cysticercosis as a real endemic trouble in the area and, overdosis of information in proper language, diffuse to the whole population, seems to be the only remedy to fight against it. Moreover, we should pay special attention to everyone related to swine farming, does not matter how this activity has been classificated.

KEY WORDS: neurocysticercosis, epilepsy, swine farming.

Neurocisticercose (NC) é a expressão usada para designar a infecção do sistema nervoso pelo Cysticercus cellulosae, forma larvária da Taenia solium. O número de cisticercos pode variar de 1 a

*Professor da Disciplina de Neurologia da Universidade Federal de Santa Catarina (UFSC); **Médico Sanitarista da Fundação Nacional de Saúde; ***Doutorando do Curso de Medicina da UFSC. Aceite: 11-outubro-1997.

Dr. Paulo Cesar Trevisol Bittencourt - Disciplina de Neurologia, Hospital Universitário UFSC - 88040-900 Florianópolis SC - Brasil. FAX 048 2431349. E-mail: pcb.latino@ intergate.com.br 
500 ou mais, assim como o tamanho dos parasitos, que podem medir menos de $1 \mathrm{~cm}$ até 6-7 ou mais centímetros de diâmetro (cistos gigantes). Sintomas neurológicos poderão advir em consequência de: efeito mecânico de pressão dos cisticercos sobre as estruturas nervosas; bloqueio da circulação liquórica por oclusão do sistema ventricular pelos cistos ou por reação inflamatória meníngea; destruição de tecido nervoso por reação inflamatória parenquimatosa ou por infarto isquêmico secundário a vasculite ${ }^{3,8,15}$. Após um período variável, provavelmente mais de 5 anos, os cistos morrem e degeneram. Fibrose residual e calcificação são o resultado final da morte e degeneração do cisticerco no parênquima cerebral ${ }^{14}$. Descrita originalmente por Aristóteles no século $\mathrm{V} \mathrm{AC}^{15}$, a cisticercose tem adquirido importância crescente nas últimas décadas. Típica de países subdesenvolvidos porém não exclusiva destes, NC é enfermidade insultuosamente frequente no Brasil, gerando grande dispêndio financeiro para o atendimento das distintas complicações neurológicas relacionadas a ela. Nosso país possui diversas áreas endêmicas, sendo a região sul considerada uma área de alto risco ${ }^{12}$. Tal fato, muito provavelmente, deve-se às peculiaridades da nossa região, com saneamento básico deficiente e que tem na suinocultura uma das mais tradicionais atividades econômicas do meio rural.

O presente estudo é parte de um esforço epidemiológico para dimensionar a magnitude dos problemas ocasionados por $\mathrm{NC}$ em nossa sociedade, objetivando auxiliar na erradicação deste flagelo terceiro-mundista, que tantas vítimas produz em nossa pátria. Trata-se de investigação pioneira realizada numa região do nosso Estado, que tem na suinocultura uma das bases de sustentação econômica. Seus resultados deverão servir de base para futuras comparações assim como para analogias com estudos similares feitos em outras regiões do nosso Estado e do Brasil.

Objetivos - Geral: Estabelecer a prevalência de NC como provável etiologia de epilepsias sintomáticas em uma região catarinense que tem na agro-indústria a principal base de sustentação econômica. Específicos: Estabelecer qual o percentual da população internada pelo diagnóstico de epilepsia na região que evidencie acometimento tomográfico por essa condição; sugerir estratégias para o controle de ambas, NC e epilepsia secundária.

\section{MATERIAL E MÉTODOS}

Foi feito estudo retrospectivo de casos de internação por epilepsia no Hospital Regional de Chapecó (HRC), região oeste do Estado de Santa Catarina. A investigação cobriu todas as internações ocorridas no período entre o início do funcionamento da tomografia computadorizada no ano de 1995, até o final do mês de novembro96. Procurou-se estabelecer a etiologia da epilepsia, através da análise individual dos prontuários de todos os pacientes internados por esta condição neste período. Todas as internações por crises febris foram excluídas.

Após levantamento inicial, os pacientes foram divididos da seguinte forma: a) pacientes sem TC no prontuário; b) pacientes com TC positivas para NC; c) pacientes com TC negativas para NC.

Todos aqueles que apresentaram evidências radiológicas de NC como provável causa da epilepsia foram identificados e estudados separadamente. Critérios diagnósticos tomográficos foram aqueles sugeridos por Sotelo et al. ${ }^{14}$. Alguns poucos pacientes tinham sido submetidos previamente a TC ambulatorialmente; entretanto, como o exame não estava disponível para análise, todos estes foram desconsiderados para fins deste estudo.

Para se estabelecer uma prevalência aproximada de NC, levou-se em consideração somente aqueles pacientes com TC disponíveis para análise. Nós partimos do pressuposto que a cifra permaneceria nesse padrão caso conseguíssemos TC em toda a amostra.

\section{RESULTADOS}

No período compreendido entre a entrada em atividade da TC em 95 e o mês de novembro de 96, cem (100) pacientes foram internados devido ao diagnóstico de epilepsia no HRC. Analisando os prontuários desses casos, chegamos ao seguintes resultados: 44 casos com TC negativa para NC; 42 casos sem TC; 14 casos com diagnóstico firmado de NC através da TC. Entre os casos positivos para NC, 6 apresentavam lesões em atividade e 8 calcificações típicas de fase crônica.

Prevalência aproximada levou em conta o número daqueles pacientes com laudo da TC no prontuário. Esses correspondem ao total de 58 casos. Como o número de pacientes com laudo positivo para neurocisticercose é 14 , encontramos a expressiva taxa de $24 \%$. 
Tabela. Distribuição por faixa etária dos pacientes com NC.

\begin{tabular}{cccc}
\hline Faixa Etária & Frequência & Porcentagem & Porcentagem acumulada \\
\hline 10 a 19 & 2 & 14,3 & 14,3 \\
20 a 29 & 3 & 21,4 & 31,7 \\
30 a 39 & 2 & 14,3 & 50,0 \\
40 a 49 & 5 & 35,7 & 85,7 \\
50 a 59 & 2 & 14,3 & 100 \\
Total & 14 & 100 & \\
\hline
\end{tabular}

Através da análise da naturalidade e procedência dos pacientes acometidos, constatamos que 3 pacientes (21\%) são provenientes de área urbana e 11 (79\%), de zona rural. Analisando a idade deles, encontramos média de 37,36; (idade máxima: 58; idade mínima 15; desvio padrão \pm 13.99 (Tabela). Dos 14 casos, 11 eram do sexo masculino (79\%) e 3 do sexo feminino (21\%).

Por outro lado, analisando naturalidade e procedência dos indivíduos não acometidos encontramos o mesmo número entre os habitantes de zonas rural e urbana, que corresponde a 22 (total 44). A idade média corresponde a 28,12 (idade máxima 80; mínima: 2; desvio padrão $\pm 19,95$ ). Dos 44 casos, 27 eram do sexo masculino $(61,3 \%)$ e 17 (38,7\%) do sexo feminino.

\section{DISCUSSÃO}

NC é uma condição disseminada por todo o país, não configurando exagero afirmar tratar-se de uma endemia nacional; ao que tudo indica, não existe um único estado da federação livre dela. Estados nordestinos e limítrofes, antigamente considerados como isentos deste flagelo terceiromundista, devido a peculariedades climáticas, passam a exibir, com a disponibilidade da TC, taxas similares àquelas observadas pioneiramente em estados do sul ${ }^{1,6,10,16}$. Por outro lado, ao contrário do apregoado por muitos (médicos inclusive), a ingestão de carne suína contaminada com larvas viáveis, em princípio, expõe o indivíduo ao desenvolvimento de teníase e não de cisticercose. Seres humanos poderão ser contaminados por cisticercos através da auto ou hetero infecção. A auto infecção ocorre em indivíduos já portadores de teníase, quando chegam anéis maduros de tênia ao nível do estômago, cujos ovos sofrendo liberação através da ação de enzimas gástricas, disseminam-se sistemicamente pela corrente sanguínea. Isto é possível por dois mecanismos distintos, seja pelo refluxo de conteúdo intestinal (auto infecção interna), seja pela ingestão de proglotes eliminadas em suas fezes (auto infecção externa) e levados à boca por mãos contaminadas após a defecação ou ainda, por hábitos coprofágicos, como acontece não raro em doentes mentais ${ }^{3}$. A hetero infecção, provavelmente a forma mais frequente de aquisição desta condição, decorre da ingestão de água ou alimentos contaminados com ovos liberados pelos proglotes de outros indivíduos parasitados pela Taenia solium. Além disso, devido aos hábitos sexuais vigentes, uma transmissão através práticas sexuais orais, é possibilidade que não deveria ser negligenciada atualmente, principalmente quando se leva em conta os milhares de ovos expelidos diariamente por um individuo com teníase.

Como métodos complementares diagnósticos úteis, podemos citar várias alternativas. $\mathrm{O}$ mais simples deles é o exame parasitológico de fezes, permitindo a identificação de ovos de tênia nos indivíduos suspeitos de estarem infectados e infectando o meio em que vivem ${ }^{2}$. O exame de líquido céfalo-raquidiano, imprescindível em muitos casos para uma segura comprovação diagnóstica, assim como para fornecer evidências de atividade de NC, poderá exibir simultaneamente hipercitose com presença de eosinófilos e positividade de reações imunológicas específicas, como da reação de fixação de complemento ${ }^{13}$. $\mathrm{O}$ estudo radiológico simples do crânio mostra, na sua fase crônica, calcificações intracranianas com as 
características morfológicas sugestivas de cisticercos; contudo, TC constitui nos dias atuais um dos métodos mais confiáveis para a sua identificação ${ }^{11,14}$; certamente, foi a vulgarização deste método em nosso meio, que contribuiu decisivamente para termos uma noção mais exata da magnitude deste problema em nosso país. Ela, além de permitir um diagnóstico apropriado, fornece evidências em relação a atividade ou não da doença ${ }^{14}$. Pequenas calcificações arredondadas, esparsas pelo parênquima cerebral ou cerebelar são sua marca registrada naqueles em fase inativa. Além disso, poderão ser também observados nódulos maiores, arredondados, realçados pela administração de meio de contraste, muitas vezes envoltos por acentuado edema perilesional, vistos na fase ativa. TC é capaz também de detectar hidrocefalia obstrutiva, uma das complicações mais temíveis de NC.

O estudo com ressonância nuclear magnética do crânio (RNM), apresenta vantagens em relação a TC particularmente nos casos em que os cisticercos estiverem localizados nas cisternas de base, na fossa posterior e nos ventrículos. Contudo, deve ser salientado que RNM não é capaz de identificar as pequenas lesões calcificadas típicas da $\mathrm{NC}$ em sua fase crônica e, por isso, naqueles pacientes com epilepsia em que a suspeita etiológica recair sobre NC, é imprescindível a realização de TC $^{21}$. Aliás, a alta prevalência desta condição entre nós torna imperativa a realização primeira de TC, em todo paciente com epilepsia cuja etiologia é incógnita. Por outro lado, é consensual que apesar das diversas manifestações neurológicas possíveis em decorrência de NC, crises epiléticas, constituem o sintoma mais comumente associado ${ }^{8,15,16}$. Porém, ainda não são disponíveis estudos definitivos, indicando a frequência com que os indivíduos portadores da forma intraparenquimatosa de NC desenvolvam epilepsia, havendo sugestões de ser uma taxa próxima a 90\%,7,8,16. Mas, a despeito da sua alta prevalência, permanece incógnita a real porcentagem com que essa parasitose contribui na etiologia das epilepsias sintomáticas em nosso meio. Trevisol-Bittencourt, em 1993, achou NC como a mais provável etiologia em aproximadamente $10 \%$ de uma população com epilepsias diversas, proveniente de todas as regiões do Estado de Santa Catarina ${ }^{19}$. Ela certamente varia muito de região para região, podendo representar proporções expressivas naquelas comunidades situadas em zonas endêmicas.

$\mathrm{Na}$ área estudada por nós, detectamos que número significativo de pacientes internados por epilepsia apresentava evidência tomográfica de NC. Uma prevalência aproximada de $24 \%$ foi observada. Apesar de relatos anedotários prévios sugerirem a alta prevalência encontrada, nós ficamos surpresos com esta cifra. Nós sabíamos de antemão que a área em estudo tem na suinocultura uma das suas principais bases de sustentação econômica; entretanto, como ela é dirigida por agro-indústrias de projeção internacional sediadas na região, nós estimávamos que técnicas modernas irradiadas por tais empresas, desde há várias décadas, influenciassem para uma minimização deste problema. Isto parece não estar ocorrendo, haja visto que 6 dos 14 pacientes, detectados como portadores de $\mathrm{NC}$, apresentavam lesões ativas, sugestivas de infecções recentemente adquiridas. Tal fato deveria ser merecedor de atenção por parte das autoridades da área da saúde, assim como despertar a classe empresarial envolvida, para a realização de campanhas que visem ao real controle desta endemia. Devido a sua pujança econômica e crescente progresso social e educacional, nós acreditamos ser exequível uma erradicação deste problema na região; contudo, sucesso nesta empreitada, somente poderia ser aguardado, caso a mensagem educativa atinja toda a população vivendo na área, e, de maneira mais incisiva ainda, a todos aqueles envolvidos em suinocultura, independentemente de como ela seja classificada. Tal sugestão é embasada não somente pela constatação de que a grande maioria dos pacientes infectados era proveniente de áreas rurais circunvizinhas mas, também, pelo reconhecimento, desde há muitos anos, que o melhor caminho para reduzir a incidência desta condição é a interrupção do ciclo tênia/cisticerco ${ }^{13}$. A propósito, é mister reconhecer que o fundamentalismo, ainda vigente em determinadas religiões influentes, ao proibir a ingesta de carne suína aos seus fiéis, empresta grande contribuição para um não reconhecimento público do "Porcus sapiens" como o principal mantenedor desta enfermidade.

Nós gostaríamos de salientar também que nossa pesquisa, em decorrência das suas limitações metodológicas, apenas toca na ponta do "iceberg". Isto é, ela enfoca apenas uma das possíveis 
manifestações desta infecção. Certamente, epilepsia é o sintoma mais frequente de NC; porém, sintomas neurológicos mais graves poderão advir, e eles não foram considerados em nosso estudo ${ }^{5,15,17}$. Neurocirurgiões, por exemplo, constituem um dos alvos prediletos desta condição e apesar de inexistirem estatísticas, nós podemos imaginar quantas vezes foram surpreendidos por NC, operando casos previamente diagnosticados como tumor ou abscesso cerebral ${ }^{4,9}$. Aliás, alguns pacientes com epilepsia, seguidos por nós ambulatorialmente, exibem um ponto em comum em suas histórias: foram operados no passado por supostos tumores cerebrais. Na maioria deles, senão todos, o diagnóstico mais apropriado era o de NC e a cirurgia, além de dispensável, constituiu agressão adicional ao parênquima cerebral. Além disso, manifestações não neurológicas de cisticercose são por demais comuns para que possam ser ignoradas ${ }^{5,16}$. Logo, nosso estudo não exibe todo o potencial mórbido que esta condição possui.

Por outro lado, chamou nossa atenção o fato de que todos aqueles pacientes com diagnóstico de NC através TC, aparentemente, não tinham outra alternativa etiológica para as suas epilepsias mencionada em seus prontuários. Em outro estudo, recentemente realizado, encontramos $\mathrm{NC}$ em alguns pacientes com epilepsia, que apresentavam evidências clínicas sugestivas de terem suas epilepsias causadas por outras condições. Tal fato sugere que NC pode ser achado acidental e que seu diagnóstico tomográfico não deveria ser sempre assumido como a causa da epilepsia de um determinado paciente em particular, principalmente quando este apresenta história sugerindo outra possibilidade causal e/ou crises cuja classificação clínico-eletroencefalográfica não estejam em concordância com as lesões da $\mathrm{NC}^{19}$.

Em resumo, poderia ser dito que eventuais pacientes sofredores de epilepsia, com diagnóstico tomográfico de NC, terão como etiologia da sua epilepsia uma outra condição. Assim, não devemos assumir sem uma avaliação criteriosa, que a NC documentada seja a causa irrefutável da epilepsia em todos aqueles pacientes. Como não foi possível uma avaliação clínico-eletroencefalográfica da amostra, nós consideramos NC como a mais provável etiologia. Tendo em conta as precárias observações contidas nos prontuários médicos, nós admitimos a possibilidade de eventualmente um ou outro paciente positivo para NC ter sua epilepsia causada por uma outra condição incógnita.

Em termos de classificação das crises epiléticas relacionadas à NC, sabe-se que elas preferencialmente apresentam-se como parciais simples ou complexas, com ou sem generalização secundária e tendem a ocorrer em salvas. Além disso, alterações no EEG, representadas por atividade delta polimorfa intermitente ou ondas sharp repetitivas focais, são achados frequentemente associados, porém inespecíficos. Entretanto, estudos eletrencefalográficos poderão ser normais nestes pacientes. Enfatiza-se que possíveis alterações detectadas poderão ser consequências de outras causas que não NC. Epilepsias geneticamente herdadas certamente existem; todavia, em nosso país a história familial positiva para epilepsia, deveria conduzir ao raciocínio da possibilidade de "NC Familial", antes da admissão de uma origem genética do problema.

Considerando aspectos terapêuticos da epilepsia secundária a NC, admite-se que as crises podem ser bem controladas através do uso racional de qualquer uma das drogas anti-epiléticas (DAE) maiores da atualidade; contudo, taxas de recidiva das crises após supressão da DAE parecem ser mais elevadas que as observadas em outros grupos de pacientes com epilepsia. Tal comportamento parece justificar o aforisma de que a epilepsia causada por NC é de fácil tratamento mas difícil de curar ${ }^{7}$. Este é mais um detalhe que deveria contribuir para reforçar a convicção, entre profissionais da área da saúde, de que todos os esforços deveriam ser dirigidos para uma eficaz profilaxia da infecção.

Infelizmente, não conseguimos viabilizar a realização da TC em 42 pacientes devido principalmente a obstáculos burocráticos, dificeis de contornar. Entretanto, a despeito de todas as limitações encontradas durante a realização deste estudo, nós acreditamos ter logrado êxito em atingir nossa meta: revelar a importância de NC na gênese das epilepsias apresentadas pelos pacientes da região oeste de Santa Catarina. 
Lamentavelmente, nossos dados são similares aos já descritos por outros autores enfocando diferentes áreas do nosso país ${ }^{16}$. É bastante provável que NC seja a principal responsável pela exagerada e vergonhosa prevalência de epilepsia na sociedade brasileira, estimada entre $1-2 \%$ da população em geral $^{20}$. Em razão disso, nós acreditamos que a criação de centros regionais voltados exclusivamente para o atendimento de pacientes com epilepsia, exerceria um papel fundamental na luta para um efetivo controle desta infecção no Brasil ${ }^{18}$. Além disso, o reconhecimento de $\mathrm{NC}$ como uma endemia nacional, torna imperiosa a adoção da notificação compulsória por todos os estados da federação. Desnecessário, por óbvio, discorrer sobre a urgência de tal medida no enfrentamento desta condição; e todos os profissionais da área da saúde deveriam estar cientes da sua importância. A nós médicos, independendo da especialidade e, não somente pela respeitabilidade social que ainda ostentamos, mas principalmente por sermos o receptáculo das tragédias provocadas por NC, caberia o ônus da iniciativa e do bom exemplo.

Em conclusão, com base nos dados levantados, nós podemos concluir que a região de Chapecó, oeste de Santa Catarina, a despeito do seu notável progresso econômico, apresenta uma exagerada e vergonhosa prevalência de neurocisticercose.

Agradecimentos - As seguintes pessoas foram fundamentais para a realização deste estudo e por isso são merecedoras dos nossos mais sinceros agradecimentos: a sra. Elmy Anshau, assessora da direção do HRC; o Dr. Antônio Carlos E. Marasciulo, epidemiologista do Hospital Universitário/UFSC e o Dr. Rogério Filomeno Machado, Coordenador Regional da Fundação Nacional de Saúde/SC. Financiamento parcial por parte da Fundação Nacional de Saúde, PNUT, projeto BRA-93015, deve também ser reconhecido como essencial para a viabilização desta pesquisa.

\section{REFERÊNCIAS}

1. Albuquerque ES, Galhardo I. Neurocisticercose no Estado do Rio Grande do Norte. Arq Neuropsiquiatr 1995;53:464-470.

2. Arruda WO, Camargo NJ, Coelho RC. Neurocysticercosis: an epidemiological survey in two small rural communities. Arq Neuropsiquiatr 1990;48:419-424.

3. Arruda WO, Meneses MS, Antoniuk SA. Neurocisticercose: diagnóstico e tratamento. Rev Bras Neurol 1996;32:15-20.

4. Arruda WO, Ramina R, Pedrozo AA, Menezes M. Brainstem cysticercosis simulating cystic tumor lesion. Arq Neuropsiquiatr 1994;52:431-434.

5. Bruck I, Antoniuk SA, Wittig E, Accorsi A. Neurocisticercose na infância: diagnóstico clínico e laboratorial. Arq Neuropsiquiatr 1991;49:43-46.

6. Chequer RS, Vieira VLF. Neurocisticercose no estado do Espírito Santo. Arq Neuropsiquiatr 1990;48:431-440.

7. Del Brutto $\mathrm{OH}$. Prognostic factors for seizure recurrence after withdrawal of antiepileptic drugs in patients with neurocysticercosis. Neurology 1994;44:1706-1709.

8. Del Brutto OH, Santibañes R, Noboa CA, Aguirre R, Diaz E, Alarcon TA. Epilepsy due to neurocysticercosis: analysis of 203 patients. Neurology 1992;42: 389-392.

9. Gallani NR, Zambelli HJL, Roth-Vargas AA, Limoli C Jr. Cisticercose medular. Arq Neuropsiquiatr 1992;50:343-350.

10. Gonçalves-Coelho TD, Coelho MDG. Neurocysticercosis in Paraiba, northeast Brazil: an endemic area? Arq Neuropsiquiatr 1996;54:565-570.

11. Machado LR, Nóbrega JPS, Barros NG, Livramento JA, Bacheschi LA, Spina-França A. Computed tomography in neurocysticercosis: a 10 year long evolution analysis of 100 patients with an appraisal of a new classification. Arq Neuropsiquiatr 1990;48:414-418.

12. Ministério da Saúde, Fundação Nacional da Saúde (FUNASA). Projeto para o controle do complexo teníase/cisticercose no Brasil. Brasília, 1996:9-14.

13. Spina-França A, Livramento JA, Machado LR. Cysticercosis of the central nervous system and cerebrospinal fluid: immunodiagnosis of 1573 patients in 63 years (1929-1992). Arq Neuropsiquiatr 1993;51:16-20.

14. Sotelo J, Guerrero V, Rubio F. Neurocysticercosis: a new classification based in active and inactive forms: a study of 753 cases. Arch Intern Med 1985; 145:442-446.

15. Stokes H. Aspectos relevantes de neurocisticercosis. Correo de Ciencias Neurologicas da Guatemala 1989:90;1:21-24,

16. Takayanagui OM, Castro e Silva AAMC, Santiago RS, Odashima NOS, Terra VC, Takayanagui AMM. Notificação compulsória da cisticercose em Ribeirão Preto -SP. Arq Neuropsiquiatr 1996;54:557-564.

17. Takayanagui OM, Jardim E. Aspectos clínicos da neurocisticercose: análise de 500 casos. Arq Neuropsiquiatr 1983;41:50-63.

18. Trevisol-Bittencourt PC. Dez razões para a criação de um Centro Catarinense de Epilepsia. Arq Cat Med 1988;17:175-176.

19. Trevisol-Bittencourt PC. Redução de drogas em pacientes com epilepsias refratárias à politerapia anti-epilética. Tese de Mestrado em Medicina Interna, Universidade Federal de Santa Catarina, Florianópolis, 1993.

20. Trevisol-Bittencourt PC, Pozzi CM, Becker N, Sander JWAS. Epilepsia em uma Instituição Psiquiátrica. Arq Neuropsiquiatr 1990;48:261-269.

21. Trevisol-Bittencourt PC, Sander JWAS. Epilepsia do lobo temporal. J Liga Bras Epilep 1991;4:27-30. 